\title{
Analysis on Separation and Purification of Corosolic Acid and Antitumor
}

\author{
Activity \\ Shucai Liang ${ }^{1, a}$ \\ ${ }^{1}$ Luohe Medical College, Luohe, Henan, China, 462000 \\ a email
}

Keywords: Loquat Leaf, Corosolic Acid, Separation and Purification, MTT Method

\begin{abstract}
Objective: To make a study of the separation and purification of purified corosolic acid from loquat leaf extract using the traditional Chinese medicine technology and the separation process in different samples of human gastric carcinoma BGC823 cell growth inhibition. Methods: 95\% ethanol extracts were purified by macroporous adsorption resin, silica gel column chromatography and preparative chromatography. The MTT method was used to test the effects of different samples on the growth of BGC823 in gastric cancer cells. Results: corosolic acid can inhibit the growth of gastric cancer cell line BGC823, isolated from different Coro cable acid samples with different degree of inhibition and other components (such as hawthorn acid or mature acid) and the coronation cable acid can produce synergistic effect. Conclusion: the method can provide experimental basis for the establishment of purification of corosolic acid and the development of new anticancer drugs.
\end{abstract}

The triterpenoid compound has a strong anti-inflammatory, antitussive, hypoglycemic, anticancer hepatoprotective and other kinds of biological activity, which is expected to develop into a high effective and low toxic biomedical functional product. Loquat leaf triterpene acids content is higher, the Coro and ursolic acid (UA) Urosolicacid cable acid (Corosoli acid. The CA) can be about 1 percent of the dry weight of the loquat leaf is the main characteristic components of loquat leaf. Corosolic acid, also known as 2 alpha hydroxy ursolic acids is in Lagerstroemia speciosa, loquat and other plants of a pentacyclic triterpenoid compounds and derivatives of ursolic acid and its molecular formula for $\mathrm{C} 30 \mathrm{H} 48 \mathrm{O} 4$, relative molecular weight is 472 . Recent studies have found that corosolic acid has hypoglycemic, slimming, anti-tumor, anti-inflammatory, anti-virus and anti-cardiovascular and cerebrovascular diseases, with very broad application prospects. Cancer is a common and frequently occurring disease which seriously threatens human life, and its mortality is only second after cardiovascular disease. And in recent years while the study of a large number of anti-tumor drugs, but the current clinical application of chemical drugs often appear side effects and adverse reactions of various on the human body, resulting in tumor patients in the course of treatment increased the new medicine source disease cannot adhere to treatment forever. Therefore, searching for effective antitumor drugs from natural products has become a hot research topic at home and abroad.

This experiment purified different corosolic acid samples via the separation purification method separated from the $95 \%$ ethanol extract of loquat leaf. We found that the samples of gastric inhibited cancer BGC823 cell growth to different degrees. It provided experimental basis for the establishment of purification of corosolic acid and the development of new anticancer drugs. 


\section{Experiment}

Experimental Instrument. The of USD 4580, JONAN SA CO2 (France), Furniture incubator (Shanghai Chinese) Taiwan aseptic operation, XDS-1B type inverted microscope (Chongqing), YXOSG41280 type portable pressure steam sterilizer (Shanghai Medical Nuclear Instrument Factory), Z323K (Germany), BCD-290WGBN refrigerated centrifuge type refrigerator (Korea S connected MSUNG company) VXE380, -80 (French Joudan) C refrigerator, CMR8030 tank (United States), LX-100 (palm type centrifuge Haimen Jiangsu BEI'ER the instrument manufacturing Co. Ltd), MS1 KA (Shanghai Mini oscillator and Science Instrument Co., Ltd.), DKB-501A super constant temperature water tank (Shanghai Jinghong Experimental Equipment Co., Ltd.) BS124S, electronic balance (sartorius Ag), AP-99015 type vacuum pump (Tianjin autoscience Instrument Co. Ltd.), E-1312-EC1-MIOST digital PH (Shanghai xunyin Commerce \& Trade Co., Ltd.), HRDG-80A electric heating air blast drying chamber (Qingdao Haier Group Co., Ltd.), II EASYpure ultrapure water meter (Barnstead). F129004A Ex-pert DNase standard instrument (Austria TECAN company)

Experimental Materials and Reagents. Coro cable acid, hawthorn acid and ursolic acid standard products (by the China drugs and biological products assay), human gastric carcinoma cell line BGC823 (purchased from cell bank of Chinese Academy of Sciences, Shanghai) were cultured in DMEM base, calf serum, fetal bovine serum (FBS), BSA bovine serum albumin egg white, trypsin, penicillin, streptomycin and HEPES powder was purchased from hyclone), MTT and DMSO was purchased from sigma company), cell counting plate, cell scraping son, cell culture bottles, 96 hole plate, 24 pore plate (Costar America).

Experimental Methods. Chromatographic conditions: Determination of acid, cable hawthorn acid and ursolic acid in samples with high performance liquid chromatography at. Chromatographic conditions: Yilite hypersil ods2 column (diameter 6 4. X 250mm. 4 m); temperature of 30 DEG C. Mobile phase was methanol - 1.0\% acetic acid solution (volume ratio of 88: 12); detection at 210nm; flow rate of $1.0 \mathrm{ml} . \mathrm{min}-1$. Sample weight $20 \mu \mathrm{L}$; corosolic acid standard as a reference.

Loquat leaf different Coro cable acid sample preparation: Firstly, ethanol extraction: take a certain amount of loquat leaf powder said, placed in a round bottom flask, adding the $95 \%$ ethanol solution, under the condition of 80 DEG C reflux extraction, extracting 2 times $2 \mathrm{H}$. The extraction solution was added to the active carbon decolorization, filtration, and acid precipitation as the extract (S1) Secondly, under the condition of $\mathrm{pH}=11$. Macroporous adsorption resin enrichment and purification: extract with $35 \%$ ethanol solution adjusted to $\mathrm{pH}=11$ dissolved prepared a certain concentration of sample solution in 1BV/h over FD16 resin enrichment and purification, by different concentrations of ethanol distribution elution, solvent recovery are beige powder namely Coro cable acid crude products (S2). Thirdly, silica gel column chromatography purification: take just the right amount of Coro cable acid crude product by silica gel column chromatography, with ethyl acetate as eluent, the collected HPLC determination of Coro cable of the upper part of the acid purity, solvent recovery was white powder namely Coro cable acid purified (S3). Fourth, preparative chromatography purification by preparative column chromatography purification, mobile phase of methanol: $1 \%$ acetic acid aqueous solution with: 10.Prepare under the wavelength of $210 \mathrm{~nm}$. Collect corosolic acid outflow liquid. Recover the solvent extract and gain the white powder as the corosolic acid products (S4).

MTT assay was used to detect the cell proliferation inhibition rate: the logarithmic growth of gastric cancer cells after tryptic digestion inoculation in 96 well plates, cell culture to completely adherent and exchange degree to $70 \sim 80 \%$. The experimental group each hole added containing DMEM, and blank control group. All groups were set up six wells in 37 DEG C, $5 \% \mathrm{CO}_{2}$ incubator 
culture for a certain time after by adding $10 \mu \mathrm{L}$ MTT solution each hole, in 37 DEG C, 5\% $\mathrm{CO}_{2}$ incubator to culture for 4 hours. After the end of the training, gently draw the supernatant in the hole (Note: cannot scrape off the cell), in each hole to join $80 \mu$ IDMSO. The hole wrap after oscillation in table 10min, faster. After the end of the oscillation, the enzyme standard instrument in $490 \mathrm{~nm}$ at the optimum wavelength of was measured, and the inhibition rate was calculated as follows: the inhibition rate $=$ control group - treatment group $* 100 \%$,

\section{Results and Discussion}

Preparation Results of Different Coro Cable Acid Sample Using Loquat Leaf. We use the above preparation methods of different Coro cable acid samples of HPLC determination of corosolic acid purity and other components of purity.

Inhibitory Effects on BGC823 Cells of Different Concentrations of Corosolic Acid. At different concentration detected by MTT acid (S4), 0.005 cable 0.01, 0.02, 0.03, 0.04, 005 mg-mL1.The inhibition of BGC823 cells was treated at different time (24 h, $36 \mathrm{~h}, 48 \mathrm{~h}$ ), and the results were shown in Figure 1. The results showed that when the corosolic acid concentration is 0.005 $0.05 \mathrm{mg}$ - ML - 1 range, cell inhibition rate with corosolic acid concentration increased and increased and increased with incubation time prolonged, corosolic acid inhibition effect on BGC823 cells in a dose - and time-dependent manner; when the concentration is $0.005 \sim 0.1 \mathrm{mg}-\mathrm{ml}-1$ range, corosolic acid effect on BGC823 cells, 24h, 36h, 48h inhibited rate difference. When the concentration of $0.01 \sim 0.04 \mathrm{mg}-\mathrm{ml}$ of the range, with the increase in the rate of increase in the rate of increase is more obvious. When corosolic acid concentration. $05 \mathrm{mg}-\mathrm{ml}-1$, in BGC823 cells at $24 \mathrm{~h}$ and $48 \mathrm{~h}$. The inhibition rates were $64.5 \%$ and $76.7 \%$ respectively. The study found that corosolic acid has high inhibition on BGC823 cells in $0.05 \mathrm{mg}$ - $\mathrm{ml}-1$ concentration rate.

Inhibitory Effects on BGC823 Cells of Different Corosolic Acid Samples. The S1, S2, S3, S4 four different Coro cable acid samples prepared corosolic acid concentrations were 5, 10, 20, 30, 40, $50 \mu \mathrm{g}-\mathrm{ml}-1$ solution. MTT cell toxicity was detected after BGC823 cell 24h, and the inhibition rate was calculated. Data showed that when the corosolic acid concentration are the same, inhibition rate of size S1 > S2 > S3, S4, suggesting that varieties of other groups (such as hawthorn acid and ursolic acid) and the coronation cable acid synergistically generate results. Different samples of BGC823 cell inhibition rate with the corosolic acid concentration increase and increase, and showed that the inhibitory effect of four kinds of samples of BGC823 cells showed a dose dependent. But S1 and S2 on BGC823 cell inhibition rate in corosolic acid concentration was $20 \mathrm{~g}-\mathrm{ml}-1$ and the inhibitory rate increased slightly with increasing concentration; corosolic acid concentration in 20 $40 \mathrm{~g}-\mathrm{ml}-1$, four different corosolic acid samples of inhibition of difference in the rate of large, corosolic acid concentration lower $(<20 \mathrm{~g}-\mathrm{ml}-1)$ or higher $(>40 \mathrm{ug}-\mathrm{ml}-1)$, the inhibition of four kinds of sample rate difference is very low. Therefore, different Coro isolated cable acid samples of gastric cancer cell line BGC823 growth have different degrees of inhibition, and other groups divided (mountain hawthorn acid and ursolic acid) and the coronation cable acid in corosolic acid concentration is 20 to $40 \mathrm{~g}-\mathrm{ml}$ - 1 to generate great synergistic effect.

Morphological Observation. The normal and tumor cells were polygonal and larger under the inverted microscope. The nuclear volume accounted for about two-thirds of the total cell volume cell aggregation and adherent growth. Different Coro cable acid samples after $24 \mathrm{~h}$ of cell rounding, volume was small and significantly reduced the number of cells, most cells breaking off and floating in the culture medium. It indicated the corosolic acid samples can effectively inhibit the proliferation of tumor cells. 


\section{Conclusion}

This experiment purified the corosolic acid from the loquat leaf using 95\% ethanol and gained the different purity corosolic acid samples by macroporous adsorption resin, silica gel column chromatography and its preparation chromatography. The MTT method was used to detect effects of corosolic acid on growth of gastric cancer cells BGC823. The results showed that corosolic acid inhibition effect on BGC823 cells in a dose - and time-dependent manner, and corosolic acid in 0.05mg-ml-1 concentration under the condition of gastric cancer cells with high inhibition rate. Effect of detection of different samples of corosolic acid on growth of gastric cancer cells BGC823 by MTT method. Results showed that isolated different corosolic acid samples had inhibition effects of different degrees on gastric cancer cell line BGC823 growth. When the other components (such as mountain hawthorn acid and ursolic acid) and the coronation cable acid in corosolic acid concentration is 20 to $40 \mu$ g- ml-1, they can produce great synergy.

\section{Acknowledgements}

This research was financially supported by the Foundation of Fundamental and Frontier Plan Project of Henan Provincial Department of Science and Technology in 2013 (Grant No. 132300410466).

\section{References}

[1] Yang Qingxin, Huang Jianan, Liu Zhonghua, Science and Technology of Food Industry, Vol. 15 (2008) No 3, p.282-285

[2] Chen Jianfeng, Chen Hao, Guo Yanghao, Chinese Patent NO. ZL200610069441. 2

[3] Chen Jianfeng, Chinese Patent NO. ZL200810071064. 5

[4] Guo Shihua, Lou Hongxiang, He Yanli, World Phytomedicines, Vol. 20 (2008) No 5, p.190-196 